Article original

\title{
Effet de la durée de conservation sur la qualité du pollen en production de semences chez le cocotier (Cocos nucifera L.)
}

\author{
Saraka D. M. YAO ${ }^{1,2}$, Konan J. L. Konan ${ }^{2 *}$, Raoul S. SiÉ ${ }^{1}$, Ablan R. Assa $^{3}$ \& Kouassi Allou ${ }^{2}$ \\ ${ }^{1}$ Université d'Abobo-Adjamé, UFR des Sciences de la Nature, Laboratoire de Biologie et d'Amélioration des Productions \\ Végétales, 02 BP 801 Abidjan 02 (Côte d'lvoire). \\ ${ }_{2}^{2}$ Centre National de Recherche Agronomique, Station de Recherche Marc Delorme, Laboratoire de Fécondation Artificielle sur le \\ programme cocotier, 07 BP 13 Abidjan 07 (Côte d'lvoire). Tel : + 22505174183 \\ ${ }^{3}$ Université de Cocody, UFR Biosciences, Laboratoire de Biochimie et Sciences des Aliments, 22 BP 582 Abidjan 22 (Côte d'Ivoire). \\ *Auteur pour les correspondances (E-mail : konankonanjeanlouis@yahoo.fr) \\ Reçu le 07-01-2010, accepté le 06-05-2010.
}

\section{Résumé}

Les présents travaux visent à proposer des voies d'optimisation du rendement de la production de semences par fécondation contrôlée chez le cocotier. Les variétés de cocotier Grand de Comores (GCO) et Nain Jaune de Malaisie (NJM) ont été utilisés à cet effet. Le pollen produit à partir de l'inflorescence est conditionné sous vide et conservé au congélateur à $-15^{\circ} \mathrm{C}$ pendant 4 mois. Chaque mois, la qualité du pollen est évaluée. II ressort que GCO fournit par inflorescence 8,6 g de pollen et NJM, 5,7 g. Pendant la conservation les taux de nouaison diminuent en général de 20,5 à $1,1 \%$ après 4 mois. Les teneurs en protéines et en sucres non réducteurs diminuent respectivement de 30 à $7,1 \%$ et de 7,9 à 2,3 \% alors que celles des sucres réducteurs augmentent de 0,7 à 1,1\%. La qualité du pollen conservé au delà de 1 mois est donc altérée et affecte le rendement de la production de semences.

Mots clés: Cocotier, fécondation contrôlée, qualité du pollen, conservation.

\begin{abstract}
Effect of the storage time on the quality of pollen in the seeds production at the coconut palm (Cocos nucifera L.).

The main objective of this study is to find the ways to optimize the yield of controlled pollination in the coconut palm. Pollen from the coconut varieties Comoro Moheli Tall (CMT) and the Malayan Yellow Dwarf (MYD) stored in deepfreezer for 4 months at $-15^{\circ} \mathrm{C}$ is studied. This study shows that CMT and MYD produced $8.6 \mathrm{~g}$ and $5.7 \mathrm{~g}$ of pollen per inflorescence, respectively. During the conservation of pollen the seed-set rate decreases from 20.5 to $1.1 \%$ after 4 months. The protein rate deacreases from 30 to $7.1 \%$. The non reducing sugars rate decreases from 7.9 to $2.3 \%$ but reducing sugars rate increases from 0.7 to $1.1 \%$. This work reveals that the quality of pollen after the first month of storage is altered and affects the outputs of controlled pollination.
\end{abstract}

Key words: Coconut palm, controlled pollination, quality of pollen, storage. 


\section{Introduction}

Le cocotier est composé de deux variétés naturelles réunies au sein de la seule espèce Cocos nucifera L. On distingue les « Grands » qui sont en général allogames et les «Nains » qui sont autogames (Sangaré et al., 1978). Le cocotier est une plante monoïque. L'inflorescence est une spadice constituée d'un rachis central sur lequel s'insèrent de nombreux épillets qui portent dans leur partie basale, des fleurs femelles surmontées de nombreuses fleurs mâles (Konan et al., 2008). La durée de la floraison mâle est d'environ 20 jours tandis que celle de la floraison femelle varie de 3 à 5 jours chez les variétés allogames et peut atteindre 15 jours chez les autogames (Sangaré et al., 1978). Le cocotier est cultivé sur 12 millions d'hectares comme oléagineuse dans les zones tropicales humides du monde. En Côte d'Ivoire, la cocoteraie couvre 50000 ha avec une production de 55000 tonnes de coprah $a^{-1}$ (Konan et al., 2006). La nuciculture fait vivre plus de 20000 familles sur le littoral (Assa et al., 2006).

De façon générale, la productivité des cocoteraies est faible (Assa et al., 2006). Une telle faiblesse est due au non respect des itinéraires techniques, aux ennemis et ravageurs du cocotier et au matériel végétal non performant planté en milieu paysan. Pour améliorer la production du cocotier, une collection constituant un réservoir important de gènes pour les programmes de sélection variétale a été établie en Côte d'Ivoire (Frémond \& De Nuce, 1971). L'introduction de variétés exotiques a commencé en 1955 (Frémond \& De Nuce, 1971). La collection de cocotier en Côte d'Ivoire couvre 1200 ha et est riche de 99 accessions. Ce qui vaut l'érection de ce patrimoine depuis 1999 en germoplasme international de cocotier pour l'Afrique et l'Océan Indien (Konan, 2005). Aujourd'hui les accessions plantées au champ deviennent de plus en plus âgées. La haute taille des arbres limite l'accessibilité de leurs inflorescences pour les travaux de fécondations contrôlées préalables à la production de semences légitimes. Ainsi, les programmes d'amélioration variétale et d'approvisionnement en matériel végétal des pays qui en font la demande sont compromis. La régénération des accessions initialement introduites apparaît donc nécessaire afin de disposer continuellement des arbres au champ pour les besoins de recherche et du développement. Depuis 1988, un programme de régénération des accessions âgées d'au moins 20 ans a été initié. La méthode consiste à produire de façon artificielle des semences de descendants qui seraient génétiquement identique aux parents par la technique de fécondation contrôlée (Konan et al., 2008 ; Yao, 2008). Elle nécessite l'apport du pollen d'un géniteur mâle identifié, sur les fleurs femelles réceptrices d'une plante mère en condition isolée. L'isolement se fait autour de l'inflorescence préalablement émasculée de la plante mère à l'aide d'un sac perméable à l'air mais empêchant la pénétration du pollen indésirable. Le rendement en semences issues de telles fécondations demeure faible. En effet, seulement 0 à $10 \%$ des fleurs femelles traitées par régime arrivent à maturité et donnent des noix (De Nuce et al., 1980). Les causes de ces faibles rendements pourraient être la qualité du pollen, le stress biotique ou abiotique subit par les arbres et/ou l'influence du microclimat autour de l'inflorescence. Les présents travaux visent à optimiser les rendements en semences issues des fécondations contrôlées chez le cocotier par l'évaluation des quantités de pollen produites et leur fertilité au cours de la conservation.

\section{Matériel et méthodes}

\subsection{Matériel végétal}

L'étude porte sur le pollen issu de deux variétés de cocotier que sont le Nain Jaune de Malaisie (NJM) et le Grand de Comores (GCO). Ces deux variétés sont sur la liste des vielles accessions à régénérer de la collection internationale pour l'Afrique et l'Océan Indien hébergée par le Centre national de recherche Agronomique (CNRA). Cette collection est maintenue à la station Marc Delorme $\left(5^{\circ} 14,5^{\prime}\right.$ de latitude Nord et $3^{\circ} 54,5^{\prime}$ de longitude Ouest). Un effectif de 45 arbres âgés de 25 à 38 ans est choisi au sein de chaque variété dont 15 géniteurs mâles et 30 plantes mères. Les géniteurs mâles retenus comme source de pollen ne sont pas utilisés comme plante mère. Le NJM et le GCO sont plantés respectivement à la densité de 205 et 143 arbres ha-1. 


\subsection{Méthodes}

Quinze inflorescences fraîchement ouvertes sur les géniteurs mâles qui n'avaient pas d'antécédent d'attaque de maladie et de ravageur sont échantillonnées de façon aléatoire par variété. Une inflorescence est sélectionnée par arbre. Les opérations de récolte des fleurs mâles au champ et d'obtention du pollen au laboratoire suivent la technique décrite par De Nuce et al. (1980).

\subsubsection{Quantité de pollen produite}

La quantité de pollen produite par inflorescence est évaluée pour les 15 géniteurs mâles échantillonnés par variété. Les masses de fleurs mâles fraîches et séchées à l'étuve à $40^{\circ} \mathrm{C}$ pendant $24 \mathrm{~h}$ sont déterminées à l'aide d'une balance électronique de marque SARTORIUS de précision $10^{-2} \mathrm{~g}$. De même, la masse de pollen extraite des fleurs mâles séchées issues d'une inflorescence est évaluée. Les rendements en pollen par inflorescence sont calculés en faisant le rapport de la masse de pollen par inflorescence et la masse de fleurs mâles fraîches ou séchées.

\subsubsection{Taux de nouaison, de germination et} caractéristiques biochimiques du pollen

Trois lots de 5 inflorescences de différents arbres sont constitués aléatoirement sur l'ensemble des 15 géniteurs mâles échantillonnés par variété. Le lot résulte du mélange homogène de la quantité de pollen, obtenue sur l'ensemble des 5 inflorescences. Soit un total de 6 lots de pollen (3 lots par variété) pour les deux variétés. Pour le conditionnement, chaque lot de pollen est subdivisé et conservé dans des ampoules en verre fermées avec un bouchon en caoutchouc, sous vide à l'aide d'une pompe à vide. Pour chaque variété et par lot, 25 unités d'ampoules sont conditionnées. Selon les quantités de pollen nécessaires pour les différents tests de qualité et la durée mensuelle de conservation $(0,1,2,3$ et 4 mois), le nombre d'unités d'ampoules a varié comme suit : 10 ampoules de $0,25 \mathrm{~g}$ pour la détermination au champ du taux de nouaison, 5 ampoules de $0,25 \mathrm{~g}$ de pollen pour la mesure $\mathrm{du}$ taux de germination in vitro, 5 ampoules de $0,25 \mathrm{~g}$ pour l'évaluation de l'humidité et 5 ampoules de $3,5 \mathrm{~g}$ de pollen pour les autres dosages biochimiques. Au total, 75 unités d'ampoules de pollen sont conditionnées pour chaque variété et conservées à $-15^{\circ} \mathrm{C}$ au congélateur. A l'issue des différentes durées de conservation, une moyenne sur les 3 lots de pollen est déterminée pour chaque variable de qualité par variété étudiée.

Le pouvoir fécondant du pollen est fourni par les taux de nouaisons obtenus en fécondations contrôlées 3 mois après pollinisation selon la méthode décrite par De Nuce et al. (1980). Les semences obtenues à partir de l'opération sont de type : GCO x GCO et NJM x NJM. Pour chaque variété et à un temps de conservation donnée, on réalise des fécondations contrôlées sur 6 arbres. Au terme des 4 mois de conservation, 30 arbres sont utilisés comme plante mère pour chaque variété. La germination in vitro du pollen est évaluée suivant le protocole établit par De Nuce et al. (1980).

Au niveau des caractéristiques biochimiques du pollen, le taux d'humidité est déterminé par pesée du poids frais et du poids sec du pollen (séché à l'étuve pendant $24 \mathrm{~h}$ à $104^{\circ} \mathrm{C}$ ) à l'aide d'une balance électronique de marque SARTORIUS de précision $10^{-2} \mathrm{~g}$. Le taux d'humidité est calculé suivant la formule proposée par ISTA (2005). L'extraction des lipides est réalisée par la méthode du soxhlet classique selon la norme ISO/659. Les autres dosages sont effectués sur le pollen déshuilé et l'expression finale des résultats est ramenée en gramme pour $100 \mathrm{~g}$ de pollen initial. Les protéines du pollen sont dosées par la méthode Kjeldhal. Les teneurs en sucres totaux et réducteurs sont évaluées par la méthode de l'acide 3,5 dinitro-salicylique (DNS) (Dubois et al., 1965). Les teneurs en sucres non réducteurs sont déduites par différence entre celles des sucres totaux et réducteurs.

\subsubsection{Analyses statistiques}

Une analyse de variance (ANOVA) à un facteur (temps) est effectuée pour évaluer l'effet du temps de conservation du pollen de chaque variété sur sa qualité. Le test de Newman-Keuls est ensuite utilisé pour faire des comparaisons intravariétales. En outre, des tests $t$ de Student sont réalisés afin de faire des comparaisons intervariétales. Des coefficients de corrélations ( $r$ ) sont calculés pour mesurer le lien entre les différentes variables de qualité du pollen pendant sa conservation. Les analyses sont effectuées à l'aide du logiciel SPSS 16.0 pour Windows (SPSS, 2007). 


\section{Résultats}

\subsection{Quantité de pollen produite}

La variété GCO produit $8,6 \mathrm{~g}$ de pollen par inflorescence. Cette valeur est statistiquement supérieure à la production de $5,7 \mathrm{~g}$ par inflorescence enregistrée chez NJM (Tableau 1). En moyenne une inflorescence de cocotier produit $400,9 \mathrm{~g}$ de fleurs mâles fraîches qui séchées pèsent $164,2 \mathrm{~g}$. Une masse de $7,1 \mathrm{~g}$ de pollen est obtenue en moyenne par inflorescence. Par rapport à la masse de fleurs mâles sèches, le rendement en pollen est de 4,6\% chez NJM et $5,2 \%$ chez GCO. Suivant la masse des fleurs mâles fraîches, le rendement en pollen est 1,7 $\%$ et $2,1 \%$ respectivement chez NJM et GCO. Les différences observées ne sont pas significatives. Pour toutes les variables étudiées, le cœfficient de variation est plus élevé chez GCO que chez NJM (Tableau 1).

Tableau 1: Quantités de pollen produites chez deux variétés de cocotier

\begin{tabular}{lcccc}
\hline \multirow{2}{*}{ Variables étudiées } & \multicolumn{2}{c}{ Variétés } & \multirow{2}{*}{ P inter-variétal } & \multirow{2}{*}{ Moyenne } \\
\cline { 2 - 3 } MacO & NJM & & \\
\hline CV (\%) & 329,2 & 372,7 & 0,622 & 400,9 \\
\hline Masse de fleurs mâles séchées (g) & 185,2 & 143,3 & 0,446 & \multirow{2}{*}{164,2} \\
CV (\%) & 47,8 & 40,1 & & \\
\hline Masse de pollen (g) & 8,6 & 5,7 & 0,018 & 7,1 \\
CV (\%) & 23 & 17,5 & & \\
\hline Rendement en pollen / frais (\%) & 2,1 & 1,7 & 0,321 & 1,9 \\
CV (\%) & 19,8 & 13,1 & & \\
\hline Rendement en pollen / sec (\%) & 5,2 & 4,6 & 0,543 & 4,9 \\
CV (\%) & 28,7 & 26 & & \\
\hline
\end{tabular}

C.V : Coefficient de Variation

Rendement en pollen / frais : Rendement en pollen par rapport à la masse de fleurs mâles fraiches ;

Rendement en pollen / sec : Rendement en pollen par rapport à la masse de fleurs mâles séchées.

\subsection{Taux de nouaison}

Le taux de nouaison passe de $20,5 \%$ au temps initial ( 0 mois) à 1,2\% au 4 ème mois de conservation chez GCO (Tableau 2). II régresse de 14,2 à 1,1\% respectivement de 0 à 4 mois chez NJM. La baisse enregistrée chez NJM n'est pas significativement différente. Chez GCO la baisse du taux de nouaison est significative après le temps initial de conservation et reste statistiquement identique entre 1 et 4 mois. Après 3 mois de conservation, le taux de nouaison chez $\operatorname{NJM}(3,7 \%)$ est significativement supérieur à celui du GCO $(1,2$ $\%)$. En moyenne, on enregistre chez les deux variétés de cocotier un taux de nouaison à 0 mois
$(17,3 \%)$ statistiquement supérieur celui relevé à 1 mois $(5,3 \%), 2$ mois $(4 \%), 3$ mois $(2,4 \%)$ et 4 mois $(1,1 \%)$ de conservation (Tableau 2$)$.

\subsection{Taux de germination in vitro du pollen}

Chez GCO, le taux de germination passe de 39,8 $\%$ au temps initial (0 mois) à 27,6 \% après 4 mois de conservation (Tableau 2). La germination du pollen baisse de 40,1 à 29,7\% chez le NJM respectivement de 0 à 4 mois de conservation. La baisse du taux de germination pendant la conservation du pollen est significative au sein de chaque variété. Une différence intervariétale significative est observée à 1 mois de 
conservation où la germination du pollen de NJM $(36,6 \%)$ est statistiquement supérieure à celle du GCO (33,3 \%). En moyenne, la germination du pollen des deux variétés confondues, diminue significativement de 39,9 (temps initial) à $29 \%$ après 4 mois (Tableau 2).

Tableau 2 : Variation des taux de nouaison et de germination du pollen et durant la conservation chez deux variétés de cocotier

\begin{tabular}{|c|c|c|c|c|c|}
\hline \multirow[b]{2}{*}{ Variables } & \multirow[b]{2}{*}{$\begin{array}{c}\text { Durée de } \\
\text { conservation du } \\
\text { pollen (mois) }\end{array}$} & \multicolumn{2}{|c|}{ Variétés } & \multirow[b]{2}{*}{$\mathrm{P}$ inter-variétal } & \multirow[b]{2}{*}{ Moyenne (\%) } \\
\hline & & GCO (\%) & NJM (\%) & & \\
\hline \multirow{5}{*}{ Nouaison } & 0 & $20,5 \pm 13,6 a$ & $14,2 \pm 8,8 \mathrm{a}$ & 0,664 & $17,3 \pm 10,1 a$ \\
\hline & 1 & $2,9 \pm 5 \quad b$ & $7,7 \pm 0,9 a$ & 0,119 & $5,3 \pm 1,5 b$ \\
\hline & 2 & $1,5 \pm 1,3 b$ & $6,5 \pm 5,6 a$ & 0,209 & $4 \pm 2,6 b$ \\
\hline & 3 & $1,2 \pm 0,2 b$ & $3,7 \pm 0,5 a$ & 0,002 & $2,4 \pm 1,4 b$ \\
\hline & 4 & $1,2 \pm 2,2 b$ & $1,1 \pm 1,9 a$ & 0,925 & $1,1 \pm 1,8 b$ \\
\hline \multirow[t]{3}{*}{$\mathrm{P}$ intra-variétal } & & 0,039 & 0,467 & 0,006 & 0,039 \\
\hline & 0 & $39,8 \pm 3,1 \mathrm{a}$ & $40,1 \pm 2,7 a$ & 0,906 & $39,9 \pm 2,6 a$ \\
\hline & 1 & $33,3 \pm 1,5 b$ & $36,6 \pm 2,8 a b$ & 0,028 & $34,9 \pm 4 \quad b$ \\
\hline \multirow[t]{3}{*}{ Germination in vitro } & 2 & $32,3 \pm 3,5 b$ & $32,9 \pm 0,6 b c$ & 0,786 & $32,6 \pm 2,2 b c$ \\
\hline & 3 & $27,6 \pm 2 \quad b$ & $31,3 \pm 2,4 \mathrm{c}$ & 0,125 & $29,4 \pm 3,8 \mathrm{c}$ \\
\hline & 4 & $28,3 \pm 1,3 b$ & $29,7 \pm 0,5 c$ & 0,151 & $29 \pm 1,1 \mathrm{c}$ \\
\hline $\mathrm{P}$ intra-variétal & & 0,001 & 0,001 & & $<0,001$ \\
\hline
\end{tabular}

Les moyennes suivies de la même lettre, dans la même colonne sont égales selon le test de Newman-Keuls (comparaison intra-variétale).

\subsection{Caractéristiques biochimiques du pollen}

Le taux d'humidité du pollen chez GCO (Tableau 3 ) est compris entre $10,4 \%$ (temps initial) et $9,4 \%$ (4 ${ }^{\mathrm{ème}}$ mois). Le pollen de NJM a un taux d'humidité qui oscille entre 7,8 et $10,9 \%$ pendant les 4 mois de conservation. II $\mathrm{n}$ y a pas de différences significatives entre le taux d'humidité enregistré au cours de la conservation chez les deux variétés. Les taux d'humidité moyens de $9,8 \%$ (temps initial), 11,2\% (1 mois), 10,1\% (2 mois), 8,8\% (3 mois) et $8,6 \%$ (4 mois) sont statistiquement identiques (Tableau 3).

Les teneurs en lipides du pollen (tableau 3 ) sont comprises entre 9,1 ( $2^{\text {ème }}$ mois) et $10,6 \%$ (3 $3^{\text {ème }}$ mois) et entre 8,3 ( $2^{\text {ème }}$ mois) et 9,6\% (3 $3^{\text {ème }}$ mois) respectivement pour les variétés GCO et NJM pendant la conservation. Les différences entre les teneurs en lipides observées au sein de chaque variété ne sont pas statistiquement significatives. Des différences significatives entre variétés apparaissent au $3^{\text {ème }}$ et $4^{\text {ème }}$ mois de conservation. En moyenne, chez le cocotier (Tableau 3), le taux de lipides se situe entre 8,7 et $10,1 \%$ pendant la conservation.

Les teneurs en protéines du pollen (Tableau 3) diminuent significativement de 29,8 (temps initial) à $7,1 \%$ (4ème mois) chez GCO et de 30 (temps initial) à $8 \%$ (4 ${ }^{\text {ème }}$ mois) chez NJM. II n'y a pas de différence de teneur entre les deux variétés excepté à 3 mois où la teneur en protéine de NJM (13\%) est supérieure à celle de GCO $(7,1 \%)$. En moyenne, la teneur en protéines dans le pollen chez les deux variétés (Tableau 3 ) est de 29,9\% (temps initial). Cette teneur baisse significativement à partir du $2^{\text {ème }}$ mois $(21,2 \%)$ pour atteindre les plus faibles valeurs au $3^{\text {ème }}(10 \%)$ et $4{ }^{\text {ème }}(7,5 \%)$ mois de conservation.

Au niveau des sucres (Tableau 3 ), les teneurs en sucres totaux (ST) baissent de 9 à $6,4 \%$ chez GCO et de 6,5 à $3,3 \%$ chez NJM après 4 mois de conservation. De 0 à 4 mois de conservation, les teneurs en sucres non réducteurs (SNR) diminuent de 7,9 à $5,5 \%$ chez le GCO et de 5,8 à 2,3\% chez NJM. Par rapport à la teneur initiale, la baisse des deux types de sucres pendant la conservation, est significative chez les deux variétés. Les teneurs en ST et SNR du pollen différencient les deux 
variétés à 0,3 et 4 mois de conservation où les valeurs enregistrées chez GCO sont supérieures à celles du NJM. Les teneurs en sucres réducteurs (SR) du pollen élevées chez GCO $(0,9 \%)$ et NJM $(0,7 \%)$ à 0 mois baissent à 1 mois de conservation où les valeurs enregistrées chez les deux variétés sont $0,5 \%$. Ensuite, les teneurs en SR augmentent à partir du $2^{\text {ème }}$ mois de conservation pour atteindre au 4 ème mois $1,1 \%$ et $1 \%$ chez GCO et NJM respectivement. La différence entre les deux variétés observée au $3^{\text {ème }}$ mois de conservation est significative. En moyenne les teneurs en ST et en SNR dans le pollen diminuent respectivement de 7,7 à $5 \%$ et de 6,8 à 3,9\%. A partir du $2^{\text {ème }}$ mois de conservation, la teneur en SR du pollen augmente en moyenne de 0,5 à $1 \%$. Les variations observées sont statistiquement identiques pour les ST et significatives pour les SNR et SR (Tableau 3).

Tableau 3 : Variation des caractéristiques biochimiques du pollen durant la conservation chez deux variétés de cocotier

\begin{tabular}{|c|c|c|c|c|c|}
\hline \multirow[b]{2}{*}{$\begin{array}{l}\text { Teneur en } \mathrm{g} / 100 \mathrm{~g} \\
\text { de pollen initial }\end{array}$} & \multirow[b]{2}{*}{$\begin{array}{l}\text { Durée de conservation } \\
\text { du pollen (mois) }\end{array}$} & \multicolumn{2}{|c|}{ Variétés } & \multirow[b]{2}{*}{$\mathrm{P}$ inter-variétal } & \multirow[b]{2}{*}{ Moyenne (\%) } \\
\hline & & GCO (\%) & NJM (\%) & & \\
\hline \multirow{5}{*}{ Humidité } & 0 & $10,4 \pm 0,5 a$ & $9,2 \pm 2,5 a$ & 0,445 & $9,8 \pm 1,7 a$ \\
\hline & 1 & $11,5 \pm 3,6 a$ & $10,9 \pm 3,8 a$ & 0,837 & $11,2 \pm 3,3 a$ \\
\hline & 2 & $9,9 \pm 2,6 a$ & $10,4 \pm 5,3 a$ & 0,908 & $10,1 \pm 3,7 a$ \\
\hline & 3 & $9,5 \pm 1,3 a$ & $8,1 \pm 1,3 a$ & 0,225 & $8,8 \pm 1,4 a$ \\
\hline & 4 & $9,4 \pm 0,9 a$ & $7,8 \pm 1,3 a$ & 0,149 & $8,6 \pm 1,3 a$ \\
\hline \multirow[t]{3}{*}{$\mathrm{P}$ intra-variétal } & & 0,738 & 0,467 & & 0,401 \\
\hline & 0 & $10 \pm 0,6 a$ & $9,3 \pm 0,9 a$ & 0,344 & $9,6 \pm 0,8 a$ \\
\hline & 1 & $9,7 \pm 1,7 a$ & $8,9 \pm 1,5 a$ & 0,575 & $9,3 \pm 1,5 a$ \\
\hline \multirow[t]{3}{*}{ Lipides } & 2 & $9,1 \pm 1,2 \mathrm{a}$ & $8,3 \pm 0,4 a$ & 0,330 & $8,7 \pm 0,9 a$ \\
\hline & 3 & $10,6 \pm 0,2 a$ & $9,6 \pm 0,5 a$ & 0,028 & $10,1 \pm 0,6 a$ \\
\hline & 4 & $9,4 \pm 0,3 a$ & $8,6 \pm 0,4 a$ & 0,046 & $9 \pm 0,5 a$ \\
\hline \multirow[t]{3}{*}{$\mathrm{P}$ intra-variétal } & & 0,440 & 0,422 & & 0,130 \\
\hline & 0 & $29,8 \pm 0,1 a$ & $30 \pm 1,4 a$ & 0,809 & $29,9 \pm 0,8 a$ \\
\hline & 1 & $29,5 \pm 1,01 \mathrm{a}$ & $29,1 \pm 1,4 a$ & 0,065 & $29,3 \pm 1,8 a$ \\
\hline \multirow[t]{3}{*}{ Protéines } & 2 & $18,8 \pm 1,5 b$ & $23,8 \pm 0,4 b$ & 0,523 & $21,2 \pm 8,3 b$ \\
\hline & 3 & $7,1 \pm 0,6 \mathrm{c}$ & $13 \pm 2,5 c$ & 0,016 & $10 \pm 3,6 c$ \\
\hline & 4 & $7,1 \pm 0,1 \mathrm{c}$ & $8 \pm 0,9 d$ & 0,155 & $7,5 \pm 0,7 \mathrm{c}$ \\
\hline \multirow[t]{3}{*}{$\mathrm{P}$ intra-variétal } & & $<0,001$ & $<0,001$ & & $<0,001$ \\
\hline & 0 & $9 \pm 0,6 a$ & $6,5 \pm 0,3 a$ & 0,010 & $7,7 \pm 1,4 a$ \\
\hline & 1 & $7,9 \pm 0,02 b$ & $6,2 \pm 2,3 a$ & 0,360 & $7 \pm 2 a$ \\
\hline \multirow[t]{3}{*}{ Sucres totaux } & 2 & $7,2 \pm 0,3 b c$ & $6,3 \pm 0,5 a$ & 0,077 & $6,2 \pm 0,6 a$ \\
\hline & 3 & $6,4 \pm 0,4 b c$ & $4,7 \pm 0,2 a$ & 0,028 & $5,5 \pm 0,9 a$ \\
\hline & 4 & $6,7 \pm 0,8 c$ & $3,3 \pm 0,2 b$ & 0,005 & $5 \pm 1 a$ \\
\hline \multirow[t]{3}{*}{$\mathrm{P}$ intra-variétal } & & 0,002 & 0,045 & & 0,056 \\
\hline & 0 & $7,9 \pm 0,7 a$ & $5,8 \pm 0,6 a$ & 0,013 & $6,8 \pm 1,5 a$ \\
\hline & 1 & $7,3 \pm 0,1 \mathrm{ab}$ & $5,7 \pm 2,5 \mathrm{a}$ & 0,320 & $6,5 \pm 1,8 a$ \\
\hline \multirow[t]{3}{*}{ Sucres non réducteurs } & 2 & $6,5 \pm 0,5 a b$ & $5,5 \pm 0,5 a$ & 0,229 & $6 \pm 0,5 a$ \\
\hline & 3 & $5,9 \pm 0,5 b$ & $4,1 \pm 0,2 \mathrm{a}$ & 0,006 & $5 \pm 1 a b$ \\
\hline & 4 & $5,5 \pm 0,6 b$ & $2,3 \pm 0,7 b$ & 0,005 & $3,9 \pm 1,8 b$ \\
\hline \multirow[t]{3}{*}{$\mathrm{P}$ intra-variétal } & & 0,002 & 0,031 & & 0,016 \\
\hline & 0 & $0,9 \pm 0,2 a b$ & $0,7 \pm 0,1 a b$ & 0,192 & $0,8 \pm 0,2 \mathrm{ab}$ \\
\hline & 1 & $0,5 \pm 0,1 b$ & $0,5 \pm 0,3 b$ & 0,837 & $0,5 \pm 0,2 b$ \\
\hline \multirow[t]{3}{*}{ Sucres réducteurs } & 2 & $0,7 \pm 0,1 \mathrm{ab}$ & $0,4 \pm 0,2 b$ & 0,063 & $0,5 \pm 0,1 b$ \\
\hline & 3 & $0,9 \pm 0,09 a b$ & $0,6 \pm 0,05 a b$ & 0,007 & $0,7 \pm 0,1 \mathrm{ab}$ \\
\hline & 4 & $1,1 \pm 0,2 a$ & $1 \pm 0,3 a$ & 0,608 & $1 \pm 0,2 \mathrm{a}$ \\
\hline P intra-variétal & & 0,018 & 0,032 & & 0,001 \\
\hline
\end{tabular}

Les moyennes suivies de la même lettre, dans la même colonne sont égales selon le test de Newman-Keuls (comparaison intra-variétale). 


\subsection{Corrélations entre les variables de qualité du pollen}

Plus le temps de conservation du pollen est long, moins bien le pollen germe in vitro $(r=-0,78)$ et les taux de nouaisons enregistrés sont faibles $(r=$ $0,56)$. La germination in vitro du pollen varie proportionnellement avec la teneur en protéines $(r$ $=0,70)$, et le taux de nouaison $(r=0,73)$. On enregistre une corrélation positive entre la teneur en protéines et le taux de nouaison $(r=0,53)$ ainsi que les sucres non réducteurs $(r=0,52)$. La teneur en protéines du pollen est corrélée négativement avec la teneur en sucres réducteurs $(r=-0,52)$ (Tableau 4).

Tableau 4 : Corrélations significatives entre les différentes variables afférents à la qualité du pollen conservé de deux variétés de cocotier.

\begin{tabular}{lcc}
\hline Variables corrélées & Coefficient de corrélation $(r)$ & $P$ \\
\hline Temps/TG & $-0,78$ & $<0,001$ \\
Temps/TN & $-0,56$ & 0,001 \\
Temps/PROT & $-0,89$ & $<0,001$ \\
Temps/ST & $-0,53$ & 0,002 \\
Temps/SNR & $-0,58$ & 0,001 \\
TG/TN & $+0,73$ & $<0,001$ \\
TG/PROT & $+0,70$ & $<0,001$ \\
TN/PROT & $+0,54$ & 0,005 \\
PROT/ST & $+0,50$ & 0,028 \\
PROT/SNR & $+0,52$ & 0,014 \\
PROT/SR & $-0,52$ & 0,014 \\
ST/SNR & $+0,98$ & $<0,001$ \\
\hline
\end{tabular}

TG : Taux de Germination in vitro

TN : Taux de Nouaison

PROT : Taux de protéine

ST : Taux de Sucres Totaux

SNR : Taux de Sucres Non Réducteurs

SR : Taux de Sucres Réducteurs

\section{Discussion}

Les résultats relatifs à la quantité de pollen produite montrent que la variété Grand de Comores (GCO) produit plus de pollen par inflorescence que le Nain Jaune de Malaisie (NJM). Les différences observées entre les Grands et les Nains proviendraient du fait que la première variété de cocotier produit de longs épillets portant un nombre relativement élevé de fleurs mâles que la seconde variété. La forte production de pollen par inflorescence des cocotiers Grands par rapport aux Nains révélée dans la présente étude corrobore celle observée par Nazayao et Malasaga (1989). Les masses de fleurs mâles, de pollen et les rendements en pollen varient plus au sein de la variété GCO que chez NJM. L'origine de la forte hétérogénéité observée chez GCO résiderait dans le mode de reproduction allogame qui caractérise les variétés de cocotier Grand. Par contre chez les variétés de cocotier Nain les individus sont moins diversifiés parce qu'elles sont autogames (Konan et al., 2007).

Concernant les nouaisons, le pollen conservé pendant 1 mois fournit des taux de nouaison plus faibles que celui utilisé directement. Ce résultat s'expliquerait par le traitement sévère subit par le pollen de cocotier depuis son extraction jusqu'à sa conservation qui aurait provoqué son infertilité. Ceci affecterait les nouaisons au champ chez le cocotier (Rognon \& De Nuce, 1973; Nazayao \& Malasaga, 1989). Jusqu'à 4 mois la nouaison baisse significativement chez GCO alors qu'il est relativement stable chez NJM. Le NJM serait prédisposé à produire plus que le GCO. N'Cho et al. (1988) ont montré que les Nains sont plus producteurs que les Grands. L'effet dépressif de la qualité du pollen conservé sur les nouaisons serait donc fonction de la variété. De même, les résultats de la présente étude montrent que les taux de nouaison au champ sont fortement dépendants de la germination in vitro et des modifications des teneurs des constituants biochimiques du pollen conservé.

Le taux de germination in vitro du pollen à l'état initial est en moyenne de 40,1\% chez les variétés de cocotiers étudiées. Ce taux est supérieur au seuil minimal international de qualité fixé à $35 \%$ (De Nuce et al., 1980). Les résultats obtenus montrent que le pollen du NJM se conserve plus longtemps. Ils révèlent par conséquent que ses propriétés biologiques se dégradent moins rapidement que chez GCO. En effet, Charrier (1990) a montré que l'aptitude à germer du pollen conservé diffère d'une variété à une autre.

Le suivi mensuel des teneurs en constituants biochimiques du pollen indique que les taux d'humidité initiaux, sont supérieurs à la norme de qualité qui est comprise entre 4 et $8 \%$ (De Nuce et al., 1980). Ce qui expliquerait que les échantillons de pollen conservés pour les 
fécondations contrôlées auraient subi une dégradation favorisée par l'humidité. En effet, d'après Verdeil et Pannetier (1990), une teneur en humidité supérieure à $6 \%$ du pollen de cocotier lors de l'extraction et durant la conservation favoriserait la dégradation enzymatique des substrats endogènes essentiels qui provoque l'émission du tube pollinique. La forte teneur en eau du pollen, restée constante pendant la conservation, a probablement servi de solvant dans les réactions chimiques qui se serait produites essentiellement avant la conservation et en proportion moindre pendant le stockage au congélateur. De même les résultats indiquent une relative constance de la teneur en lipides du pollen qui pourrait traduire l'inaltération de la membrane des microspores ou bicouche phospholipidique pendant la conservation. Ce résultat est concordant avec celui de Rowley (2001) qui a révélé la résistance à la dégradation de la structure de l'exine du pollen. Par ailleurs, les teneurs en sucres non réducteurs et en protéines du pollen baissent tandis que la teneur en sucres réducteurs augmente. Les variations observées de ces teneurs s'expliqueraient par la présence accidentelle d'oxygène dans les ampoules conservées qui aurait provoquée une oxydation des métabolites essentiels et réduit ainsi la vigueur du pollen (Webbert, 1996).

Concernant la corrélation entre les variables de qualité du pollen conservé, les résultats révèlent une corrélation positive entre les teneurs en protéines et en sucres non réducteurs avec la germination in vitro du pollen. D'abord, l'existence d'une corrélation positive entre les protéines et les sucres non réducteurs suggère que les deux caractères seraient soumis à une influence commune qui modifierait simultanément leurs teneurs. En effet, l'hydrolyse des sucres non réducteurs et des protéines libérerait de l'énergie (ATP) qui servirait à la germination du pollen (Mangenot, 1973). Ensuite, les produits résultant de ces mécanismes seraient des sucres réducteurs qui proviendraient de l'hydrolyse des sucres non réducteurs sous l'activité des enzymes comme l'ont mentionné Adjahossou et Viera (1978) chez le palmier à huile. De même, la forte activité enzymatique pendant le temps d'exposition du pollen à température ambiante, lors des manipulations, avant la conservation du pollen pourrait être à l'origine de toutes ces réactions d'hydrolyse et en particulier de celles qui auraient augmenté la teneur initiale en sucres réducteurs. Par contre, pendant la conservation du pollen au froid, l'activité des enzymes hydrolysant les sucres non réducteurs serait partiellement réduite. Ce phénomène s'expliquerait par les faibles corrélations négatives obtenues entre les teneurs en protéines et en sucres réducteurs.

Des corrélations positives sont obtenues d'une part, entre le taux de nouaison, la teneur en protéines et d'autre part avec le taux de germination in vitro du pollen pendant la conservation. Concernant le taux de germination in vitro du pollen, ce résultat est concordant avec celui de Digonnet et Gay (1990) qui a montré que ce facteur serait un indice de fécondité. L'influence de la baisse des teneurs en sucres non réducteurs et en protéines sur les rendements en semences pourrait s'expliquer par la faible teneur en glucides endogènes du pollen conservé qui perturberait l'émission et l'allongement du tube pollinique. Le faible pouvoir fécondant du pollen pendant la conservation pourrait également s'expliquer par des perturbations au niveau des phénomènes de reconnaissance pollen-stigmate, conséquence de la perte des glycoprotéines qui imprègnent l'exine (Charrier, 1990).

\section{Conclusion}

L'objectif de ce travail était d'optimiser les rendements en semences des fécondations contrôlées par l'évaluation des quantités de pollen produites et leur fertilité au cours de la conservation. Les caractéristiques du pollen conservé pendant 4 mois des variétés de cocotier Grand de Comores (GCO) et Nain Jaune de Malaisie (NJM) sont étudiées au laboratoire et au champ. II ressort que la variété GCO fournit plus de pollen par inflorescence $(8,6 \mathrm{~g})$ que le NJM $(5,7 \mathrm{~g})$. Les taux de nouaison observés après fécondations réalisées avec du pollen non conservé varient de 14,2 à $20,5 \%$. Après 1 mois de conservation, le taux de nouaison (2,9\%) chute significativement chez GCO contrairement au $\operatorname{NJM}(7,7 \%)$. Cette étude a permis de comprendre que la fertilité du pollen conservé est tributaire du taux d'humidité initiale et des modifications de ses teneurs en constituants biochimiques telles 
les sucres et les protéines. Pendant la conservation du pollen, les teneurs en protéines et en SNR régressent respectivement de 30 à $7,1 \%$ et de 7,9 à $2,3 \%$ alors que celles des SR augmentent de 0,7 à $1,1 \%$.

Par conséquent, il est conseillé d'utiliser le pollen conservé pendant un temps relativement court c'est-à-dire 1 mois maximum pour optimiser de plus de $10 \%$ les rendements en semences des fécondations contrôlées. Par ailleurs, les manipulations pendant la production de pollen à température ambiante devront s'effectuer rapidement afin de minimiser les réactions biochimiques qui pourraient dégrader le pollen.

\section{Remerciements}

Les auteurs remercient les responsables de Global Crop Diversity Trust (GCDT) et de International Coconut Genetic Resources Network (COGENT) pour avoir financé le projet de régénération de 50 accessions âgées de la collection internationale de cocotier du CNRA. De même, ils adressent leurs remerciements à l'équipe du laboratoire de Fécondation Artificielle de la station Marc Delorme pour sa contribution à ce travail.

\section{Références citées}

Adjahossou F. \& Viera D., 1978. Teneur en glucide soluble et en amidon et résistance à la sécheresse chez le palmier à huile. Oléagineux 33 (12) : 599-604.

Assa R., Konan K., Nemlin J., Prades A., Agbo N. \& Sie R., 2006. Diagnostic de la cocoteraie paysanne du littoral ivoirien. Sci. Nat. 3 (2) : 113-120.

Charrier A., 1990. Pollen et ressources génétiques. Bull. Soc. Bot. Fr. 137 (2) : 101-104.

De Nuce L., Wuidart W. \& Sangaré A., 1980. La fécondation artificielle du cocotier. Oléagineux 35 (4) : 319-326.

Digonnet K. \& Gay G., 1990. Qualité du pollen : définition et estimation. Bull. Soc. Bot. Fr. 137 (2): 97-100.

Dubois M., Gilles K., Hamilton J. K., Rebers P. A. \& Smith F., 1965. Colorimetric methods for determination of sugars and related substances. An. Chem. 28: 350- 356.

Frémond Y. \& De Nuce L., 1971. Le bloc d'amélioration du cocotier de port-bouet. Oléagineux 26 (2): 71-82.

ISTA, 2005. International Rules for Seed Testing (Edition 2005). International Seed Testing Association, Bassersdorf, Suisse.

Konan J.L., 2005. The International Coconut Genebank for Africa and Indian Ocean (Côte d'Ivoire). In: Batugal P., Ramanatha V. \& Oliver J., Eds. International Plant Genetic Ressources Institute. Serdang, Selangor DE, Malaysia. pp. 121-122.

Konan J.L., Allou K., N'Goran A., Diarrasouba L. \& Ballo K., 2006. Bien cultiver le cocotier en Côte d'Ivoire. Fiche technique sur le cocotier. Direction des programmes de Recherche et de l'Appui au Développement, CNRA, Côte d'Ivoire. 4 pp.

Konan J.L., Bourdeix R. \& George M.L., 2008. Directives pour la régénération : cocotier. In: Dulloo M.E., Thormann I., Jorge M.A. \& Hanson J., Eds. Crop specific regeneration guidelines [CD-ROM]. CGIAR / SGRP, Rome, Italy. 11 pp.

Konan J.N., Konan J.L., Koffi K., Lebrun P. \& Sangaré A. 2007. Coconut Microsatellite gene diversity analysis technology transfer to Côte d'Ivoire. Biotechnology 3: 383-388.

Mangenot G., 1973. Données élémentaires sur l'Angiosperme. Anales de l'université d'Abidjan, Côte d'Ivoire. 233 pp.

Nazayao L. \& Malasaga E., 1989. Male flowers and pollen characteristics of some coconut dwarf populations in the Philippines. Phil. J. Coco. S. 14 (2) : 2-6.

N'Cho Y., Le Saint J-P. \& Sangaré A., 1988. Les cocotiers Nains à Port-Bouet (Côte d'Ivoire) III.- Nain Brun Nouvelle-Guinée, Nain Vert Thaïlande et Nain Rouge Polynésie. Oléagineux 43 (2) : 55-64.

Rognon F. \& De Nuce L., 1973. Action du froid sur la conservation du pollen de cocotier. Oléagineux 28 (12): 565-566.

Rowley R., 2001. Why the endexine and ectexine differ in résistance to oxidation. Calluna as a model system. Grana 4: 159-162. 
Sangaré A., Wuidart W.\& De Nucé L., 1978. Les phases mâles et femelles de l'inflorescence du cocotier. Influence sur le mode de reproduction. Oléagineux, 33 (12): 609-617.

SPSS, 2007. SPSS 16.0 for windows. SPSS Inc., 233 south wacker drive, $11^{\text {th }}$ Floor Chicago, USA.

Verdel J. L. \& Pannetier C., 1990. Optimisation des conditions de germination in vitro du pollen de cocotier (Cocos nucifera L.) pour la mise au point d'un test de viabilité. Oléagineux, 45 (4): 175-179.
Webbert J., 1996. Douglas-fir pollen management manual ( $2^{e}$ edition). B. C. Ministry of forests, Victoria, USA, Work Paper 02/1996. 91 pp.

Yao S. D., 2008. Influence de la durée de conservation du pollen de deux génotypes de cocotier (Cocos nucifera L.) sur ses caractéristiques biochimiques et son pouvoir fécondant. Mémoire de DEA, Université d'Abobo-Adjamé, Côte d'Ivoire. $53 \mathrm{pp}$. 